\title{
Editorial
}

\section{Infrared Imaging and NDT}

\author{
Rubén Usamentiaga ${ }^{1, *(\mathbb{D}}$ and Pablo Venegas ${ }^{2}$ (i) \\ 1 Department of Computer Science and Engineering, University of Oviedo, 33204 Gijón, Asturias, Spain \\ 2 Aeronautical Technologies Centre (CTA), Juan de la Cierva 1, 01510 Miñano, Spain; \\ pablo.venegas@ctaero.com \\ * Correspondence: rusamentiaga@uniovi.es
}

Citation: Usamentiaga, R.; Venegas, P. Infrared Imaging and NDT. Appl. Sci. 2021, 11, 3024. https://doi.org/ 10.3390/app11073024

Received: 25 March 2021

Accepted: 26 March 2021

Published: 29 March 2021

Publisher's Note: MDPI stays neutral with regard to jurisdictional claims in published maps and institutional affiliations.

Copyright: (c) 2021 by the authors. Licensee MDPI, Basel, Switzerland. This article is an open access article distributed under the terms and conditions of the Creative Commons Attribution (CC BY) license (https:// creativecommons.org/licenses/by/ $4.0 /)$.
Infrared thermography has become a mature and widely accepted technology with applications in many different fields, from medical to industrial. Two main approaches are employed: passive, in which the temperature of facilities and products is monitored; and active, in which materials are subjected to external thermal stimulation. These two approaches offer many different possibilities, with novel ideas appearing frequently. This Special Issue presents thirteen papers with the latest advances in infrared thermography, covering fields such as nondestructive testing, cultural heritage, or infrared image processing. This selection of high-quality papers contributes to the advancement of research and underscores the broad potential for the future.

In [1], an infrared image processing algorithm is proposed: a super-resolution reconstruction algorithm. Infrared cameras generally have very low image resolution. This greatly limits the applicability in different fields that require specific spatial sampling frequencies. Super-resolution reconstruction algorithms aim to solve this issue by creating images with increased resolution based on single or multiple low-resolution images. In this work, an adaptive dual-regularization super-resolution reconstruction algorithm is proposed. The algorithm first enhances the multiscale detail of low-resolution images. Then, reconstruction is performed using the regular terms of the double regular terms and the double adaptive parameters. Finally, subpixel convolution is applied to merge the features and generate the high-resolution images. This step uses a convolutional neural network trained with a dataset created with blurred images. The proposed procedure is compared with other methods based on several well-known metrics, such as PSNR and SSI. Results indicate that the presented method has advantages in detail information enhancement and edge preservation. Moreover, it can effectively reduce noise and improve the overall brightness of the image. Qualitative evaluation seems to indicate better results than other algorithms.

An infrared image processing algorithm is also proposed in [2]. In this case, it addresses another challenging problem in infrared thermography: the fusion between the infrared and visible images of the same scene. Fusion requires accurate matching between the images. This is a difficult computer vision problem that greatly depends on the extracted features from the images. This work proposes a feature detection and description method based on consistent edge structures of images to solve this issue. The proposed method consists of a pipeline of operations: (1) the detection of consistent edge structures in both images (infrared and visible); (2) the extraction of common features points based on the edge structures; (3) the description of the structures, including length and orientation; (4) the calculation of the correspondences between features using bidirectional matching. The performance of the proposed method is compared with previous methods on two public datasets using feature detection repeatability and feature matching accuracy. The method provides very good results on a variety of tests, outperforming previous methods in most of them. Moreover, it achieves the second-best performance for running time.

In [3], an application of active thermography is presented. In this case, it is used to estimate the thermal resistance field quantitatively. The problem is applied to layered materials, which are crucial in the manufacturing of different high-quality products nowadays. 
This challenging problem is very difficult to solve using an analytical method. In this case, the authors propose a different strategy: using a simple multilayer problem where only one resistive layer is estimated. In addition, they propose an asymptotic development method to simplify inverse processing. The paper addresses the inability of some methods, such as thermal signal reconstruction, to provide quantitative results by incorporating a calibration process base on the complete analytical quadrupole solution. The paper describes the experiments, camera configuration, and heating. The proposed procedure presents major advances, such as the possibility to address any type of multilayer material or fast inverse processing. Results indicate that the thermal resistance field can be calculated with an accuracy lower than $5 \%$ in less than $5 \mathrm{~s}$.

In [4], Dr. Sfarra et al. present an application of active thermography to the analysis of multilayer wooden cultural heritage objects. In this case, pulse-compression thermography is applied to the sample to avoid damaging the fragile object during heating. Using this technique, a very low thermal contrast is enough to highlight the defects when using the proposed image processing method. This work proposes using the Hilbert transform to increase defect detection and characterization, and a novel normalization technique based on absorbance estimated by considering a characteristic value of the compression peak. The inspection method is applied to an inlay sample created by a professional restorer. Two experiments are performed using different cameras: an uncooled camera and a middle-wave camera. Results demonstrate the success of the proposed approach, providing high SNR and defect detection capability. This approach makes the inspection of very fragile art pieces possible, which otherwise would be damaged during heating when using active thermography.

Another application of active infrared thermography to cultural heritage is presented in [5]. This work evaluates the capability of infrared thermography to differentiate between different types of historical glass. This approach can be used, for example, to distinguish previous restoration interventions. The experimental procedure is based on halogen lamps heating the samples. Measurements are performed in reflection and transmission mode. The sequence of infrared frames acquired is processed, first by calculating an average and then by subtracting this average from each frame. The resulting sequence is then analyzed using two clustering algorithms: K-means and hierarchical clustering. Results indicate the proposed inspection method differentiates between different groups of glass panes, with clustering providing more reliable results. In addition, it provides faster results while reducing time-consuming manual analysis. The conclusion is that active infrared thermography shows potential for documenting the material intervention history of leaded-glass windows during or in preparation for conservation treatments.

A study about the characterization of the lateral dimension, depth, and inclination of buried tilted rectangular heat sources is presented in [6]. The novelty of this work lies in the consideration of an arbitrary inclination of the heat source with respect to the surface of the material, a problem few works have addressed. To analyze the problem, the authors present a semianalytical expression for the surface temperature distribution produced by a rectangular heat source of any inclination. A model is fit considering two perpendicular temperature profiles and the temperature history at one position. Moreover, the optimum experimental conditions are analyzed. Synthetic data with added noise and experimental data taken with an inductive thermography setup are used for testing. Results demonstrate the capability of the proposed approach to determine the dimensions, depth, and inclination of the heat source. Further discussion about aspects to pay attention to is provided in the paper.

An application of passive thermography is presented in [7]: the detection of defects in photovoltaic plants. Inspecting photovoltaic plants represents nowadays a costly and time-consuming operation. Moreover, the efficiency and profitability of the plants greatly depend on these operations and the considered periodic maintenance procedures. Infrared thermography is used in this work to automate the inspection of these large plants. The proposed method combines infrared thermography and unmanned aerial vehicles, providing 
a fully automated approach for the (a) detection, (b) classification, and (c) geopositioning of thermal defects in photovoltaic modules. The paper describes the configuration of the mission planner, the acquisition procedure and the proposed image processing pipeline. The automated processing first undistorts the images based on a previous calibration procedure. Then, the correction of the perspective is performed in the images and segmentation is applied to detect the defects. The final step is the conversion from pixel coordinates to GPS coordinates. The proposed system is evaluated using a large dataset of images and standard metrics for classification. Results demonstrate the capability of the system to automate the inspection of large photovoltaic plants, presenting a far more cost-efficient solution than other methods.

Infrared thermography very often requires manual analysis and interpretation of the images. This is usually performed using false color mapping, which generates visual representations of the images using a specific color palette. This mapping is problematic, particularly when images contain a wide range of infrared intensities. In [8], a method is proposed to address this issue: adaptive transformation and image fusion. The goal is to transform the raw images into new images with better contrast and clear detail. The proposed approach is divided into several steps: (1) adaptive Gamma transformation is applied to the normalized image, producing multiple brightness levels; (2) each brightness image is decomposed using the multiscale guided filter; (3) enhanced images in each brightness are fused together; (4) the outliers are filtered and images fusion is performed. Testing is performed with images from different public datasets. The experiments compare the performance of the proposed methods with other well-known methods, such as CLAHE. The authors provide a qualitative and quantitative comparison. Results indicate that the proposed method has strength in the detail enhancement of high-dynamic-range infrared images, generating visually pleasing images.

In [9], a study is presented comparing the efficiency of time- and frequency-domain analysis techniques in flash thermography, a very popular inspection method using active thermography. The goal is to compare the techniques in terms of defect detectability. The comparison is performed using various carbon-fiber-reinforced polymer samples having a range of defect sizes and defect types. The research work evaluates the advantages and drawbacks of the different postprocessing techniques. In order to quantitatively compare the techniques, the contrast-to-noise ratio is used. The comparison is complemented with the defect-to-background ratio, an alternative defect detectability metric more robust to the noise level of the reference area. The results indicate that the single-bin evaluation approach in the frequency domain provided improved defect detectability than its time-domain counterpart. However, the frequency-domain results also show a higher sensitivity to nonuniform heating. A more in-depth analysis is provided in the paper about other considerations.

Another research work on pulsed thermography is presented in [10]. This work deals with a different problem: the determination of the size of defects from the resulting inspection using active thermography. The authors propose a simple yet effective image processing pipeline based on linear operations and blob analysis. Tests are performed using a PVC plate that has two flat-bottomed defects artificially fabricated at different depths. Additional tests are performed with a CFRP specimen. The proposed method offers an automated approach for defect detection, with applications to quality assurance.

In [11], another research work is presented about detection capacity in active thermography. This work proposes a novel data analysis methodology that extracts a greater amount of information from the recorded sequences by applying advanced processing techniques. The proposed work presents a novel approach for defect detection: the extracted information is synthesized into three channels that may be represented through real color images and processed by quaternion algebra techniques. The colorization of thermographic images is a well-known method that can provide enhanced defect detectability. The novelty in this work is the application of quaternion analysis to enhance the detection capability. To validate the proposed approach, synthetic data and actual experimental data are used. 
Results are quantitatively compared using different signal-to-noise ratio metrics. Compared with other methods, such as approximate polynomial sequences and their first and second derivatives as well as principal component analysis, the quaternionic processing techniques produce higher SNR values. In some real defects, the first derivative provides better results, but in general, the proposed methodology improves results compared to conventional thermographic processing. Moreover, it improves the distinction of defects due to their representation in different color shades. This work also concludes that the choice of SNR definition to assess the results has a great impact on the analysis.

An interesting industrial application of infrared thermography is presented in [12]. This work uses active infrared thermography to inspect rails. Inductive thermography is used in this case, as it can identify surface cracks very effectively. Two different approaches are considered: stop-and-go and line-scan. The former method takes several images of the rail and creates a single, combined panorama image. The latter scans the rail continuously while the rail is moving forward along the track. The two inspection approaches are analyzed and compared, underscoring the advantages and disadvantages for each of them. In addition, different image processing tools are proposed to fully characterize the rail defects. The research work also includes finite element simulations to further analyze the problem and to optimize the experiments. Testing concludes that inductive thermography is an excellent nondestructive testing method for localizing surface defects in metallic materials. Further research work is planned, but tests with different specimens show very promising results.

The last paper of this special issue presents a methodology to optimize dynamic linescan thermography [13]. The authors of this work include Prof. Maldague, one of the most prolific and influential researchers in the field of infrared thermography. Dynamic line-scan thermography is a relatively new inspection method, which requires a complex parameter tuning procedure that mostly depends on the material's properties. The research work presents an optimization approach using the response surface that can give more insights about the interconnections between parameters. Throughout the paper, the creation of the response surface is discussed and how it can be used for the optimization, offering an explanation for the influence of every variable used. Tests are performed on a PVC flat-bottom hole plate. Authors claim that the proposed optimization routine offers an insight about the working principle of dynamic line-scan thermography, providing a better understanding about the influence of the different parameters on each other.

Overall, the Special Issue includes a great selection of peer-reviewed papers covering some of the areas where infrared thermography is making more advances and impact, from industrial applications using active and passive thermography to nondestructive testing, image processing, visualization, and cultural heritage applications. Infrared thermography is a very active field with constant advances and innovations. This Special Issue presents an overview of the wide applicability of the technique, which could serve as a guideline or inspiration for further research and novel applications in the future. Moreover, this compendium of papers highlights some of the challenges and opportunities in the field, offering some promising research directions.

Institutional Review Board Statement: Not applicable.

Informed Consent Statement: Not applicable.

Data Availability Statement: Data sharing not applicable.

Acknowledgments: The authors would like to thank the talented authors and the reviewers for their excellent contributions to this Special Issue. Special thanks to Canna Ren, from the editorial team, for all the help and the support in the publication process.

Conflicts of Interest: The authors declare no conflict of interest. 


\section{References}

1. Yu, L.; Zhang, X.; Chu, Y. Super-resolution reconstruction algorithm for infrared image with double regular items based on sub-pixel convolution. Appl. Sci. 2020, 10, 1109. [CrossRef]

2. Wang, Q.; Gao, X.; Wang, F.; Ji, Z.; Hu, X. Feature Point Matching Method Based on Consistent Edge Structures for Infrared and Visible Images. Appl. Sci. 2020, 10, 2302. [CrossRef]

3. Groz, M.M.; Bensalem, M.; Sommier, A.; Abisset-Chavanne, E.; Chevalier, S.; Chulkov, A.; Battaglia, J.L.; Batsale, J.C.; Pradere, C. Estimation of thermal resistance field in layered materials by analytical asymptotic method. Appl. Sci. 2020, 10, 2351. [CrossRef]

4. Sfarra, S.; Laureti, S.; Gargiulo, G.; Malekmohammadi, H.; Sangiovanni, M.A.; La Russa, M.; Burrascano, P.; Ricci, M. Low Thermal Conductivity Materials and Very Low Heat Power: A Demanding Challenge in the Detection of Flaws in Multi-Layer Wooden Cultural Heritage Objects Solved by Pulse-Compression Thermography Technique. Appl. Sci. 2020, 10, 4233. [CrossRef]

5. Hillen, M.; Legrand, S.; Dirkx, Y.; Janssens, K.; Van der Snickt, G.; Caen, J.; Steenackers, G. Cluster Analysis of IR Thermography Data for Differentiating Glass Types in Historical Leaded-Glass Windows. Appl. Sci. 2020, 10, 4255. [CrossRef]

6. Mendioroz, A.; Fuggiano, L.; Venegas, P.; Ocáriz, I.S.d.; Galietti, U.; Salazar, A. Characterizing Subsurface Rectangular Tilted Heat Sources Using Inductive Thermography. Appl. Sci. 2020, 10, 5444. [CrossRef]

7. Fernández, A.; Usamentiaga, R.; Arquer, P.d.; Fernández, M.Á.; Fernández, D.; Carús, J.L.; Fernández, M. Robust Detection, Classification and Localization of Defects in Large Photovoltaic Plants Based on Unmanned Aerial Vehicles and Infrared Thermography. Appl. Sci. 2020, 10, 5948. [CrossRef]

8. Chen, F.; Zhang, J.; Cai, J.; Xu, T.; Lu, G.; Peng, X. Infrared Image Adaptive Enhancement Guided by Energy of Gradient Transformation and Multiscale Image Fusion. Appl. Sci. 2020, 10, 6262. [CrossRef]

9. Poelman, G.; Hedayatrasa, S.; Segers, J.; Van Paepegem, W.; Kersemans, M. An Experimental Study on the Defect Detectability of Time-and Frequency-Domain Analyses for Flash Thermography. Appl. Sci. 2020, 10, 8051. [CrossRef]

10. Yuan, L.; Zhu, X.; Sun, Q.; Liu, H.; Yuen, P.; Liu, Y. Automatic Extraction of Material Defect Size by Infrared Image Sequence. Appl. Sci. 2020, 10, 8248. [CrossRef]

11. Venegas, P.; Usamentiaga, R.; Perán, J.; Sáez de Ocáriz, I. Quaternion Processing Techniques for Color Synthesized NDT Thermography. Appl. Sci. 2021, 11, 790. [CrossRef]

12. Tuschl, C.; Oswald-Tranta, B.; Eck, S. Inductive Thermography as Non-Destructive Testing for Railway Rails. Appl. Sci. 2021, 11, 1003. [CrossRef]

13. Verspeek, S.; Gladines, J.; Ribbens, B.; Maldague, X.; Steenackers, G. Dynamic Line Scan Thermography Optimisation Using Response Surfaces Implemented on PVC Flat Bottom Hole Plates. Appl. Sci. 2021, 11, 1538. [CrossRef] 